CAHIERS DE

NARRATOLOGIE

\section{Cahiers de Narratologie}

Analyse et théorie narratives

$23 \mid 2012$

Le sujet et l'art dans la prose française contemporaine (1990-2012)

\title{
Lenteur et vision : l'empreinte du western dans le roman français contemporain
}

\section{Elisa Bricco}

\section{(2) OpenEdition}

\section{Journals}

Édition électronique

URL : http://journals.openedition.org/narratologie/6587

DOI : 10.4000/narratologie.6587

ISSN : 1765-307X

Éditeur

LIRCES

Référence électronique

Elisa Bricco, "Lenteur et vision : l'empreinte du western dans le roman français contemporain », Cahiers de Narratologie [En ligne], 23 | 2012, mis en ligne le 29 décembre 2012, consulté le 20 avril 2019. URL : http://journals.openedition.org/narratologie/6587 ; DOI : 10.4000/narratologie.6587

Ce document a été généré automatiquement le 20 avril 2019

Article L.111-1 du Code de la propriété intellectuelle. 


\title{
Lenteur et vision : l'empreinte du western dans le roman français contemporain
}

\author{
Elisa Bricco
}

1 Dans le célèbre ouvrage de Jeanne-Marie Clerc sur Littérature et cinéma ${ }^{1}$, on suppose que le roman des années ' 60 , le Nouveau Roman mais aussi d'autres expériences d'écriture comme les premiers romans de Le Clézio et de Jean Echenoz, sont imprégnés par l'esthétique cinématographique. Depuis cette époque, la liaison étroite entre les deux formes de création n'a fait que se raffermir et la perméabilité entre cinéma et littérature est désormais une réalité incontestable. Le cinéma continue de puiser des sujets et des scénarios dans le roman et le roman intègre dans ses histoires les mécanismes et les stratégies narratives du cinéma. Cet échange réciproque entre littérature et cinéma est si riche et si fréquent que chez quelques jeunes écrivains d'aujourd'hui, l'on assiste au dépassement de l'effet rebound ${ }^{2}$, c'est-à-dire que l'on est désormais bien au-delà d'une écriture ayant intégré les pratiques de la création et de la narration cinématographiques. En fait, dans le roman contemporain, il ne s'agit plus de mimer les mouvements de la caméra, de reproduire les effets de l'image projetée sur l'écran ni d'accompagner le récit d'une bande-son adéquate qui stimule des sensations variées dans l'imaginaire du lecteur, mais bien au contraire d'écrire avec le cinéma. Le cinéma est tout à fait intégré à l'écriture romanesque, avec ses stéréotypes et ses topoï, ses techniques et ses stylèmes, et cela aboutit à la composition d'un tout unique, d'un récit multi-sensoriel.

2 Cette intégration du cinéma dans le roman est assez claire, facilement perceptible et annoncée par le titre du roman récent de Patrick Chatelier, Pas le bon pas le truand (Verticales, 2010). Il s'agit d'un rapport au cinéma affiché très ouvertement par le péritexte et que l'on constate facilement pendant la lecture. C'est en fait une écriture avec le cinéma, qui n'est pas seulement cinématographique dans ses éléments constitutifs, mais qui arrive à assimiler quelques poncifs et stéréotypes du genre western : on y trouve par exemple le cow-boy, le héros solitaire, la femme fatale ou la mère de 
famille; et aussi quelques topoï: le désert, la ferme au milieu de la prairie, le saloon, le duel. De plus, ce roman intègre dans son fonctionnement et dans la progression du récit deux éléments prépondérants du western, notamment de celui tardif, à la façon de Sergio Leone : l'importance du regard et de la vision en général, et la lenteur du récit et de son développement, qui est due à une attention aux moindres détails d'une scène et de son décor, ainsi qu'au déroulement minutieux des actions, en y incluant ces préalables et ces conséquences. L'analyse du roman, qui représente la réécriture d'une scène d'un film western bien connu, conduira alors à la découverte de la manière dont le cinéma western joue un rôle matriciel pour ce texte, le façonnant et le modelant.

\section{Du film au livre : que reste-t-il?}

3 Pas le bon pas le truand de Patrick Chatelier peut être considéré comme une variation à partir de la deuxième séquence du film de Sergio Leone, Le Bon, la Brute et le Truand, sorti en salle en 1966. Il s'agit du dernier film de la trilogie de l'homme sans nom, où le seul élément en commun entre les films est la présence de l'acteur Clint Eastwood. La séquence, qui dure huit minutes et demi, a une fonction précise dans la diégèse filmique puisqu'elle introduit l'un des protagonistes du film, la Brute, et amène le spectateur au cœur de l'histoire racontée, puisque l'on y assiste à la rencontre entre Lee Van Cleef, qui interprète le rôle de Sentenza, la Brute, et ses victimes, les membres de la famille Stevens. La Brute a été payée pour trouver Stevens père (Antonio Casas) et en tirer des informations concernant un magot qui a été caché, puis mystérieusement soustrait de la cachette; il doit ensuite le tuer. Tout se déroule selon les règles du genre, et la Brute exécute son mandat pour le compte de Baker (Livio Lorenzon) sans rechigner. C'est sur ce canevas exigu que l'écrivain a construit son texte, tout en s'appuyant sur sa réalisation filmique.

4 Naturellement, la séquence filmée est construite de manière typiquement western : un cavalier solitaire arrive de nulle part, l'enfant de la famille l'aperçoit de loin, alors qu'il est à l'extérieur de sa maison et monte l'âne tournant autour du puits pour en extraire l'eau. Par le jeu du champ/contrechamp, la caméra suit les mouvements de l'enfant et du cavalier pour ensuite se fixer sur la silhouette de celui-ci qui, en s'approchant, s'agrandit progressivement, jusqu'à remplir tout l'écran en très gros plan. Le décor est typique : le désert ensoleillé, la maison blanche isolée, le silence tout autour; aucun mot n'est prononcé, c'est le montage qui compose le récit. On entend seulement le bruit des sabots de l'âne et du puits avec l'eau, et ensuite ceux du cheval avec, évidemment, la musique d'Ennio Morricone qui contribue à créer l'ambiance et à induire un certain suspense. Le visiteur entre ensuite dans la maison où l'enfant s'est déjà faufilé, et rencontre le chef de famille. Le mouvement de la caméra nous amène à suivre la scène, toujours muette, du point de vue d'un personnage à l'autre, encore par des champs/contrechamps. En silence, sans même se saluer, l'un commence à manger, l'autre fait de même, et ils se regardent intensément. Ils commencent à se parler, discutent des raisons qui ont amené là le cavalier. Enfin, après avoir obtenu les informations qu'il cherchait, celui-ci tue l'autre, puis son fils aîné qui a accouru après avoir entendu le bruit des coups de feu.

5 Ces huit minutes de cinéma ont inspiré la création de Patrick Chatelier qui a réécrit la séquence en l'insérant dans un récit où le personnage principal change : ici c'est l'idiot du village qui regarde la scène en cachette dans un cagibi de la maison des Stevens (les Butler, dans le roman) où il se trouve par hasard. Du point de vue de la construction du 
récit, il est clair que l'action de l'écrivain ne s'est pas limitée à reproduire la séquencesource, mais il a construit un récit qui l'inclut et qui n'est donc pas celle du film, bien qu'elle en reprenne les traits fondamentaux. En somme, il a ajouté le personnage de l'idiot et l'a placé en contexte: il a créé un antécédent qui motive sa présence dans la séquence-source et il a ajouté une conclusion dont l'idiot est le protagoniste. La scène se termine ainsi de manière différente par rapport au film et le texte se clôt définitivement, tout en laissant le lecteur privé d'un véritable dénouement et en proie à plusieurs doutes.

La structure du texte suit ces développements et ces ajouts. En fait, il est divisé en six parties dont la centrale, la plus longue avec une centaine de pages, reproduit la séquence filmique par variantes successives, tandis que les autres parties sont bien plus courtes. Le texte liminaire est une introduction qui permet d'établir le pacte de lecture : ce sont deux pages qui ont un pendant dans les deux dernières, la clausule; dans les deux séquences intermédiaires de longueur analogue, une dizaine de pages, l'auteur installe son récit annonçant l'arrivée de la Brute qui peut très facilement être identifiée avec la mort, puisqu'elle est même pourvue de sa faux. Il construit le personnage de l'idiot doté d'une histoire personnelle et l'installe dans une sorte de baraque noire et inhabitée, où ses amis l'ont enfermé et qui sera son point d'observation, entre autre, de la scène chez les Stevens; à la fin, un dénouement surprise le transforme en héros de la scène, et la clausule ferme le récit tout en brisant l'illusion fictionnelle dans laquelle on est tombé en nous ramenant au début du livre, lorsque nous avions commencé à suivre les aventures visuelles de l'idiot. Comme au cinéma, l'on suit les aventures des héros et à la fin seulement l'on découvre que c'était un leurre, et que rien de ce que l'on a vu ou lu n'était réel, mais uniquement le fruit de l'imagination d'un personnage.

\section{L'incipit, un texte programmatique}

7 Ainsi que dans la meilleure tradition romanesque ${ }^{3}$, le texte liminaire établit un véritable pacte de lecture parce qu'il introduit le lecteur dans l'univers de la diégèse en lui fournissant quelques informations fondamentales sur le sujet du récit, sur sa filiation par rapport au film, sur sa connexion avec l'esthétique cinématographique par la lenteur et la vision qui impriment leurs rythmes au récit.

Qu'il est beau, le monde et son écran d'images, myriades à chaque seconde, à chaque inspiration, images qui effleurent et se détachent et incisent et se répondent, toujours pareilles, toujours nouvelles, captivantes. Elles se projettent en paysage, elles forment sous le bleu du ciel une terre à histoires panoramique, un visage à embrasser sur la bouche, se dit l'idiot.

Qu'il est élégant, le cavalier solitaire descendant de la colline, sa posture, son galop tranquille - cercles du chapeau, faisceaux des pattes-d'oie, lignes de sueur aspirées sous les mâchoires. [...] Il lui faut du temps pour traverser le paysage, un temps infini comme s'il avait la terre entière à parcourir, puisque la terre lui appartient aussi loin que porte le regard. (p. 13)

Après avoir introduit le personnage focalisé et le spectacle qu'il est en train de regarder, on passe à la présentation du décor, du village et de ses habitants qui ne seront pas de véritables personnages dans le récit: ils seront seulement évoqués par l'idiot dans ses pensées plutôt qu'être des sujets agissants. La dérivation par rapport au film est évoquée ici par la description de l'approche du cavalier avec aussi l'utilisation du plan rapproché sur son visage et ses yeux. Deux informations encore concourent à parfaire cette introduction, d'abord la mention des images que l'idiot racontera par la suite («Sur la 
rivière Arlanza derrière la colline se reflètent des images ", p. 14) et enfin la déstabilisation de toute possible feintise ludique partagée $e^{5}$, en conséquence de la suspension de l'incrédulité sur laquelle se base toute lecture empathique de la fiction: «Et je suis seul, se dit l'idiot. Et il s'invente.» Comment pourra-t-on prendre au sérieux un texte fictionnel qui se propose comme tel ? Y plonger de manière naïve et crédule, si on sait dès le début que tout n'est qu'une invention? Il est clair que les fondements sur lesquels se bâtit le pacte de lecture traditionnel s'effondrent ici et c'est un regard attentif, conscient et spéculateur que cet incipit nous impose d'assumer. Cela se réalise au point que l'on pourrait considérer le texte de Chatelier comme une véritable " fiction critique ", ainsi que l'envisage Dominique Viart ${ }^{6}$. Une fiction qui fait le clin d'œil au cinéma pour en retordre les schémas constitutifs et les travailler du dedans.

\section{Focalisation et ocularisation pour un récit multifocal}

Le récit est ainsi centré sur le personnage de l'idiot en focalisation interne ou pour mieux dire en ocularisation interne ${ }^{7}$, parce que le texte emprunte au cinéma les caractéristiques de la mise en récit où le voir et le savoir ne vont pas forcément de pair. En effet, ce texte met en place un système de narration différent par rapport aux récits où la narration s'appuie sur un point de vue avec focalisation particulière : zéro, interne ou externe, ou sur toutes les trois selon les situations racontées et sur une source de l'énonciation bien déterminée aussi, ou encore sur une polyphonie de sujets énonciateurs et donc une variation des possibles focalisations. Dans Pas le bon pas le truand l'écrivain mime le récit filmique en nous présentant le protagoniste, l'idiot, en tant que sujet qui assiste aux scènes racontées et participe à celles-ci aussi, donc en focalisation interne, mais le récit n'est pas homodiégétique, et l'on perçoit donc la présence d'une entité extérieure qui raconte et en même temps compose le récit. C'est ce que François Jost appelle focalisation spectatorielle. En calquant la notion mise au point par Gérard Genette ${ }^{8}$, le critique affirme qu'au cinéma la perception de l'histoire de la part des spectateurs est omnisciente puisqu'ils perçoivent les images, les sons et tout ce que le montage, le cadrage et la prise de vue mettent en place pour créer la narration. Par le biais de ce texte, nous sommes donc comme les spectateurs de la scène que nous suivons grâce aux mots de celui qui raconte; l'idiot fait partie de ce que nous voyons et nous percevons ses pensées aussi. Cette configuration narrative est visiblement empruntée au cinéma. En plus, dans ce récit elle n'est pas stable, mais varie selon les situations racontées, à des degrés d'intensité différents, de la focalisation spectatorielle à la focalisation interne sur l'idiot.

On a vu que dans l'incipit il est fait mention du regard et de la vision d'images, la poursuite du récit construit et développe cette problématique jusqu'à la mise en question de la réalité des faits racontés. Comme la base du récit est une autre fiction, il semble que l'auteur décide de poursuivre dans la mise en place d'un leurre, puisque l'idiot invente et s'invente :

L'idiot se frotte les yeux dans son lit [...]. Il a du mal à décider quand il dort et quand il ne dort plus. Pour certains la vie est un rêve où défilent toutes les images, les histoires, les fantaisies [...]. Ils forment et reforment, ils agrémentent, corrigent prolongent en évoluant dedans, ils parcourent les sentiers de leur monde enchanté. (p. 30) 
11 Il est clair que, si l'idiot est le personnage par qui nous suivons l'histoire racontée, l'appréhension de la réalité passe par le filtre de sa vision et de sa perception et que sans doute nous ne $\operatorname{dev}(\mathrm{ri})$ ons rien croire de ce qu'il voit.

En poursuivant la lecture, le récit qui semblait linéaire bascule dans l'invraisemblable lorsque l'idiot est enfermé dans la cabane par ses amis voulant se moquer de lui, où vit le fantôme de Piripero, un trappeur mort depuis longtemps. La cabane, dépourvue de fenêtre, est une sorte de caverne, et elle est aussi très proche d'une salle de cinéma, parce que l'idiot y voit défiler des images si vraisemblables qu'il a du mal à faire la part entre le vrai et le faux: " Il a vu des choses incroyables dans cette cabane. [...] Il a encore en tête toutes sortes d'images qui défilaient, milliers d'images avec leurs formes vibrantes, collines, plaines et villes, pays qu'il ne connaissait pas, habités par toutes sortes de gens que jamais il ne connaîtra»(p.41). De plus, lorsqu'il sort de ce merveilleux cinéma improvisé, l'idiot vit dans une dimension onirique perpétuelle, où la réalité et les images vues ne font qu'un tout unique : «Quand l'un des pieds décide de s'arrêter, le second part à la découverte. Avec une telle paire il ne faudrait pas devoir échapper à une locomotive qui te fonce dessus, ni chercher à attraper un landau qui dégringole l'escalier, mais on comprendrait l'utilité d'une canne [...]» (p. 43). Voilà qu'une fois sorti de la cabane l'idiot vit comme s'il y était encore, et par un très intéressant jeu intertextuel généré par l'évocation de scènes célèbres de films inoubliables, l'auteur contribue à faire de ce texte une sorte de cinématière, comme l'a définie Sébastien Rongier ${ }^{9}$, où les deux formes de création narrative s'interpénètrent pour aboutir à un récit visionnaire et de la vision.

\section{Le récit de la vision}

Dans ce récit, la cinématière n'est pas seulement constituée par l'intertextualité, mais aussi par la prépondérance du champ lexical de la vision et du regard, qui imprime à la matière textuelle une dimension encore plus imaginaire. Le livre, qui nous avait plongé dans une dimension western, raconte aussi l'histoire d'une vision cinématographique qui bascule vers l'illusion de la réalité, puisque l'idiot, à un certain moment, crée le cinéma de sa vie et perd la capacité de distinguer le réel de l'imaginaire. Il lui arrive ainsi de participer au film qu'il est en train de visionner ou sans doute qu'il est seulement en train de construire par son imagination. Le bouleversement de l'appréhension de la réalité à partir de la vision et par le regard est total : le réel bascule dans l'imaginaire et vice-versa. Cela est dû principalement au fait que, depuis son enfance, l'idiot a commencé à être considéré comme tel, par ses amis et par les adultes, à partir du moment où il a vu dans les reflets du fleuve des images différentes de celles que voyaient ses camarades. Sa vision du monde et le regard très personnel qu'il pose sur la réalité nous accompagnent ainsi le long de la lecture, en produisant des effets de véritable projection d'images et de scènes. Par conséquent, en tant que spectateurs, nous visualisons avec lui les scènes qu'il v(o)it, comme si l'on était au cinéma, nous retrouvant souvent dans une position de « vision par derrière ${ }^{10}$ ou ocularisation spectatorielle.

L'idiot regarde la scène arrêtée - ou sans le savoir arrête-t-il la scène de son regard. Les trois Butler devant la table attendant le signal, l'inconnu à la porte attendant un signal, les mouches continuant de voler. [...] Cette lenteur lui permet de distinguer les détails, respirations, cillements, friselis. Cette lenteur grossit les moindres gestes et vibrations, elle donne une place, une densité à son rôle de voyeur, voyeur immobile comme les quatre autres, en attente de voir. (p. 70) 
L'idiot, et nous avec lui, est un voyeur : tout ce qu'il voit devient séquence et, comme dans une salle cinématographique, nous assistons à une suite de scènes qui se succèdent l'une après l'autre, dans une narration qui a une allure scandée plutôt que fluide et continue. Le contexte cinématographique de la narration est si évident que le lexique technique apparait constamment : l'arrêt sur image et la pause qui s'ensuit évoquent les séquences du film où l'on s'arrête sur les détails pour augmenter le suspense et la perception globale des éléments qui la constituent. Au point que les insectes deviennent les parties intégrantes de la séquence et que leurs plus minimes mouvements attirent l'attention du voyeur. Celui-ci devient une sorte d'œil de caméra: il joue le rôle d'un «voyeur immobile " par qui passent les scènes racontées, et aussi celui de "coin panoramique " permettant la vision globale de la partie de la réalité représentée. D’ailleurs, l'idiotvoyeur-voyant répond aux lois imposées par les héros des western à leurs enfants, c'est-àdire que, pour exister, il faut se construire une vision : « ... fabriquez-vous une vision, car c'est le propre de l'homme : posséder sa vision, fignoler sa portée, l'imposer aux autres cow-boys. » (p. 85). Le choix fondamental de l'auteur est, ici, d'opter pour la perspective visuelle de l'idiot qui accompagne donc celle du lecteur dans sa découverte du texte. Ce sont les spécificités de sa vision, ses capacités à percevoir la réalité sous des perspectives tout à fait personnelles, qui structurent le récit au point que l'on se demande si l'idiot n'est pas l'auteur lui-même qui manipule son personnage et lui donne une liberté de vision et de création absolues.

Toutefois, le regard de l'idiot n'est pas seul dans ce texte : on trouve aussi celui des autres personnages, de ceux qu'il observe depuis sa cachette. Son ami Jesse, par exemple, est le sujet focalisé dans une partie de la scène à la maison où il regarde autour de lui pour comprendre ce qui se passe lorsque la tension entre son père et la Brute monte :

Jesse se détourne de son père un peu honteux [...] Son regard s'échappe et glisse vers Carlotta [...] Le regard de Jesse rebondit sur elle, poursuit son élan vers le bout de table en hésitant soudain sur ce qu'il s'apprête à voir, ripant maladroit sur les grains de lumière mais oui, mais non, mais si : l'étranger est toujours là, à la place de l'invité, à la place de l'ami, à la place du porteur de nouvelles. (p. 86)

Nous suivons bien aisément le parcours de ce regard comme s'il vivait d'une existence autonome; ses mouvements aménagent en quelque sorte la scène, en passant d'une personne à l'autre avec son regard très personnel sur la réalité : il bouge comme la caméra jusqu'à devenir un véritable œil-caméra. Si les mouvements du regard sont très fréquents, la teneur de celui-ci est aussi parfois très dense, presque matérielle, par exemple : «Surprise, Carlotta tressaille et se raidit fusillée sous le regard» de la Brute (p. 89), et on n'a aucune peine à le croire en reconnaissant le regard, acéré comme une lame qui vous transperce, de Lee Van Cleef lorsqu'il scrute ses victimes. Le regard est aussi le moyen qui permet une perspective surplombante sur la scène de la mort de George Butler. En fait, plutôt que de raconter cette scène par la focalisation sur l'idiot, Chatelier recourt à un stratagème intéressant - il imagine la présence d'un œil surplombant la scène, et cet œil a une consistance très particulière :

Il presse la détente et la balle va se ficher dans une poutre, frôlant au passage le cercle d'une mouche qui s'écarte puis le reforme. En vol, les mouches ont l'habitude de tracer des cercles ou des carrés, bifurquant plusieurs fois pour revenir à leur point de départ et cela dessine un œil qui regarderait d'en dessus : George depuis cet œil doucement se voit mourir. (p. 115)

17 Le passage d'un regard à un autre, mais aussi la multiplication des regards et l'effet de miroir entre eux visent à reproduire une sorte de champ-contrechamp imaginaire. Cette 
multiplication des points de vue est très significative et la création de l'œil surplombant permet de bien comprendre l'utilisation de la matière cinématographique que l'auteur plie au service de l'écriture. Il s'en sert et ose même aller au-delà, à partir d'une technique de prise de vue : avec l'œil surplombant qui évoque le regard du personnage sur lui-même en train de mourir, il crée un nouveau dispositif visionnel. Ce vol des mouches représente une autre focalisation sur la scène: cela pourrait représenter le point de vue d'un être supérieur qui regarde la scène d'en haut ou bien l'œil indifférent de la mort, ou encore celui de l'esprit de George qui est désormais sorti à l'extérieur de son corps. En tout cas, c'est le point de vue d'un narrateur omniscient. Cet œil surplombant évoque peut-être aussi le voyeurisme du spectateur, de l'idiot dans notre cas, mais aussi, sans doute, celui d'un narrateur-écrivain-cinéaste, souvent mis en place par le cinéma d'Hitchcock et de Michael Powell.

La cinématière travaille ainsi le récit: de la même manière que George, Carlotta se fait tout un cinéma intérieur quand elle comprend (contrairement à ce qui se passe dans le film de Leone) qu'elle va mourir après son mari. Très traditionnellement, on apprend que « devant ses yeux se met à défiler sa vie entière » (p. 132), et la succession des scènes de sa vie passe comme si c'était un film devant ses yeux horrifiés, juste avant qu'elle ne reçoive la balle fatale. À la fin, là aussi les mouches restent les seuls êtres vivants dans la maison et c'est leur point de vue qui est mis en évidence encore une fois : « Un vol de mouches suspendu depuis quelques minutes au-dessus des reliefs du repas atterrit en douce pour zoomer sur les miettes, sans pour l'instant s'intéresser à George [...] » (p. 135).

\section{Construction en lenteur}

À côté de l'aspect visionnel, dans l'introduction, nous avons évoqué aussi la lenteur comme une des composantes de l'écriture narrative qui dérive des westerns à l'italienne. Dans Pas le bon pas le truand, cette lenteur est reproduite de plusieurs manières: par l'utilisation du plan rapproché qui permet la description minutieuse des scènes, par la mise en premier plan des détails, enfin par la construction du récit qui présente des développements hypertrophiques de séquences.

Si on voulait représenter sur un axe horizontal cette structure, on pourrait imaginer une ligne correspondant au récit des événements, tandis que des bulles surgissant sur la ligne représenteraient les développements des personnages qui divaguent, laissent aller leur esprit librement vers leur passé. Mais on pourrait aussi plus simplement imaginer les tiroirs du roman baroque qui étaient ouverts à certains moments du récit pour développer des histoires secondaires, et qui étaient refermés à la fin de ceux-ci pour reprendre le récit principal. Quoi qu'il en soit, c'est dans ces moments de la narration que Patrick Chatelier remplit les trous de l'intrigue du film, comme s'il voulait s'insérer dans des blancs entre les scènes, dans les interstices du montage et du récit cinématographique. Il en résulte que, malgré le développement du texte en parties bien définies, le déroulement de l'intrigue n'est pas tout à fait linéaire, puisqu'il est laissé libre cours aux rêveries des personnages. Ces développements visent à donner des compléments d'informations sur le contexte et sur l'histoire. Ce sont des approfondissements, des replis des personnages sur eux-mêmes et sur leur passé, que le film ne laissait pas soupçonner. Par exemple, lorsque Carlotta comprend qu'elle va mourir, et que toute son existence passe devant ses yeux, on assiste à une très longue analepse où elle revient sur sa vie d'enfant en famille, sur les motivations qui l'ont 
poussée encore adolescente dans les bras de Butler, et sur leur vie conjugale et familiale. De plus, ces approfondissements de quelques aspects du récit correspondent aussi aux changements de la perspective narrative concernant tous les personnages : c'est comme si l'auteur voulait nous montrer la même scène depuis plusieurs points de vue et que chaque sujet focalisant transposait sur la scène sa propre vision du monde et ainsi son expérience. Jesse, l'enfant de la famille, l'ancien ami de l'idiot, devient à son tour le sujet de la narration, constituant ainsi une variation sur le thème principal, celui de la visite de l'étranger et, aussi, une réflexion sur le thème de la vision : « Une vision, rendez-la moi, dit Jesse, car la sienne vacille face à cette scène dont les ressorts lui échappent [...] Jesse n'arrive plus à y voir. [...] Alors Jesse se replie sur les visages, il se concentre, il s'accroche aux expressions pour lire, pour deviner [...]» (p. 82)

Ainsi, le rythme de l'intrigue est chargé des rêveries de l'idiot et de son cinéma intérieur. En effet, il est clair qu'il distingue très mal le songe de la réalité, et au fur et à mesure que la narration progresse ce brouillage narratif s'intensifie. La scène centrale du roman, celle qui provient du film de Sergio Leone, se présente plusieurs fois sous les yeux de l'idiot. C'est l'idiot lui-même qui manipule cette scène, comme s'il avait une télécommande en main et qu'il la faisait repasser plusieurs fois sur son écran visuel, y ajoutant chaque fois quelques éléments supplémentaires ou bien changeant de perspective et d'angle d'approche. La séquence est arrêtée, elle repart et s'arrête encore, et l'idiot la voit, la vit et l'invente à tel point qu'il devient « CEil du principe : l'idiot regarde. Principe de l'œil : regarde-t-il. Depuis sa cachette, l'idiot vit toute chose par le principe de son œil. [...] Voir fait vivre songe-t-il. Voir vivre fait songer» (p. 71). La vie et la vue se superposent et l'idiot rentre de plein pied dans la vie qu'il voit. Depuis l'appentis noir dont il a fait sa cachette, l'idiot regarde la scène qui prend des contours différents selon les moments : d'abord il s'approche lentement de la maison des Butler qui lui apparaît de plus en plus clairement, et son approche physique est accompagnée de sa rêverie, tandis que le lecteur à son tour suit son mouvement qui intercalé par ses pensées. Ensuite, lorsqu'il entre dans la maison, celle-ci est vide, exceptée une table dressée qu'il regarde: "Sur la table le couvert est mis. Trois assiettes attendent, escortées de leur cuillère en bois.» (p. 54). Cette vision sollicite sa rêverie et l'idiot imagine la scène avant de la voir vraiment. Il se questionne sur le sort des habitants de la maison et son questionnement évoque ironiquement la fable des trois ours : « Trois assiettes sur la table, trois cuillères en bois et trois couteaux, trois timbales. Ne manquent plus à la scène que trois bouches, trois avalements, trois gestes à prendre. Trois personnages se font attendre dans ce décor de trois fois rien. Trois occupations ne devraient plus tarder à converger.» (p. 56). L'idiot voit alors le fils Butler à l'extérieur et aussi la scène originaire du film, dont les trois premières minutes passent devant ses yeux. Puis, il entre dans la maison et, petit à petit, il s'approche de la salle où se déroulera le drame. Son approche rassemble à celle du spectateur qui entre dans une salle de cinéma :

La porte de derrière claque sur lui et il se retrouve dans un couloir obscur. [...] Il lui faut marcher dans le noir [...]. Tout au bout du couloir une lueur sert de guide. [...] La porte atteinte, l'embrasure étire sa ligne verticale, le grand jour se devine encadré par une fenêtre proche. [...] L'idiot ouvre doucement, se fait tout petit à mesure que la lumière se déverse et un blanc aveugle balaie le noir qui voyait tout. [...] Et peu à peu [...] ça lui répond sur sa rétine, l'espace de la maison avec la mère, le père et le fils, la grande table aux trois couverts mis, le bahut, la cheminée... (p. 65-66). 
Mais à ce moment-là, la scène se fige, elle est arrêtée, comme on a vu plus haut, « les trois Butler devant la table attendant un signal, l'inconnu à la porte attendant un signal, les mouches continuant de voler.» (p. 66). Cet arrêt sur image permet au narrateur de montrer l'attente de l'idiot et de bien construire la scène, voire de la reprendre dès le début pour la raconter depuis un autre point de vue ou pour en approfondir d'autres aspects. «Scène d'intérieur figé. Quatre immobilités autour de la table, devant les restes de nourriture. Quatre plongées en soi-même avec regards de coin. Quatre suspensions malades de ne pas se briser» (p.97). Le rythme saccadé de la narration rend bien la tension qui s'est créée autour de la table et l'angoisse qui pénètre l'idiot. Il s'ennuie, il " voudrait surgir de sa cachette et hurler ce qui lui traverse le crâne » (p. 99), il « se sent étourdi, comme après avoir tournicoté. [...] L'ennui en est la cause. L'ennui fait apparaitre, invente des images à donner le vertige.» (p. 101). Ainsi l'idiot est suspendu entre la vision réelle et sa vision personnelle d'où «il ressort de plus en plus hébété, fragile comme les corps hébétés de chagrin» (p. 102). Il est désormais en proie à son délire : nous suivons les modifications de sa vue et les circonvolutions de la narration qui est devenue tout à fait invraisemblable et en même temps très captivante, parce que l'effet de suspense est bien construit, au point que nous attendons ce qui arrivera à la fin : on se demande si la séquence filmique qu'on connaît va se reproduire ou pas. Le piétinement de la narration se poursuit et le narrateur instaure un dialogue avec son personnage: «Mais si tu regardes mieux, si maintenant tu t'extirpes totalement du songe, si tu chasses les restes de vision sous la cire et le sel, tu constateras qu'en réalité quelque chose a changé.»(p.102). L'idiot doit sortir de son songe et visualiser les hypothèses sur la scène qu'il regarde, mais malheureusement l'effort est trop important et « de nouveau cela est un songe. De nouveau ce sont les visions dans ton cerveau qui mènent leur vie propre. » Le narrateur s'adresse directement à son personnage, qui se transforme en cow-boy chevauchant sur les images: " Tu vas trop vite, idiot. Tu t'emballes avec ton idiotie. Tu grimpes sur le dos des images et tu dis Hue.» Et il lui donne aussi quelques conseils : "Reviens au geste de George, le geste de George pur, le bras droit, la jambe raide, le déplacement. Si tu patientes quelques secondes il ira jusqu'au bout. Il trouvera son terme absolument unique. Il anéantira les autres possibles et toutes les songeries dans un gong. » (p. 103).

Il est clair que l'auteur a voulu écrire une histoire où l'image visuelle est incarnée par l'écriture, non seulement en tant qu'image mais en tant que mots écrits qui ont la force de provoquer des visions. La cinématière réside donc dans ce mélange de scènes perçues par des points de vue différents et multiples qui permettent de développer le récit en plusieurs couches qui viennent se superposer l'une à l'autre, ainsi que par la multiplication des focales et des cadres rendant une réalité parcellisée, multiple et complexe.

\section{Pour un texte cinématographique}

Le cinéma, et en particulier le western à l'italienne, joue un rôle matriciel dans ce récit. Les jeux de la vision, la lenteur de la narration et les stratégies de sa construction concourent à former un texte visionnaire où le cinéma est présent à tous les niveaux de la diégèse et de la création littéraire. Patrick Chatelier joue avec les stéréotypes, l'ironie sourd de ses pages où le seul hôtel mentionné s'appelle Paramount Hôtel, et où le cadre extérieur est stéréotypé à l'excès: toutes les composantes du bon western y sont 
présentes, du General Store à la diligence, du chant à la guitare aux chardons sur les routes poussiéreuses, de la sieste au duel. Par ailleurs, le lexique de la prise de vue de la caméra est entièrement exploité, au point où les mouches deviennent des co-protagonistes, les sujets focalisés qui jouent le rôle d'œil supplémentaire scrutant la scène.

Le cinéma a apporté à la littérature une nouvelle manière de multiplier les histoires racontées, de reprendre une narration et d'y intervenir pour la parfaire et l'approfondir, mais surtout il lui a permis de rendre un récit visuel et sonore, de jouer encore avec le songe et la réalité d'une manière originale, jusqu'à maintenant jamais expérimentée. En somme, il a empreint le texte de matière cinématographique comme il a empreint l'idiot d'images en Technicolor.

\section{ANNEXES}

Elisa Bricco est professeur de littérature française à l'Université de Gênes en Italie. Depuis quelques années ses intérêts de recherche portent sur les enjeux de la communication auctoriale en littérature française moderne et contemporaine, sur les caractéristiques de la fiction narrative et la poésie d'aujourd'hui, sur l'impact des TICE dans la création et la communication en littérature et dans la didactique. Elle est responsable d'une équipe de recherche pour le projet national PRIN 2009 (Bari, Gênes et Roma La Sapienza) sur Le sujet et l'art dans le roman français contemporain (1990-2010). Elle a publié des études sur Arno Bertina, François Bon, Jean Echenoz, Sylvie Germain, Emmanuel Hocquard, Pierre Michon, Valérie Rouzeau, Eugène Savitzkaya, Antoine Volodine entre autres. Parmi ses dernières publications, elle a dirigé avec Christine Jérusalem Christian Gailly : l'écriture qui sauve (2007) ; elle a coordonné le collectif Letture del romanzo francese contemporaneo, numéro spécial de la revue Nuova Corrente (II, 2009), et également Présences du sujet dans la poésie française contemporaine (1980-2008). Figurations, configurations et postures énonciatives (2012). Elle dirige le groupe de recherche de l'ARGEC (Atelier de recherches génois sur l'écriture contemporaine) http://argec.hypotheses.org.

\section{NOTES}

1. J.-M. Clerc, Littérature et cinéma, Paris, Nathan « Université », 1993.

2. F. Ivaldi, Effetto rebound. Quando la letteratura imita il cinema, Pisa, Felici Editore, 2010.

3. A. Del Lungo, L'Incipit romanesque, Paris, Seuil, « Poétique », 2003.

4. Patrick Chatelier, Pas le bon pas le truand, Paris, Verticales, 2010. C'est nous qui soulignons. Les numéros de pages entre parenthèses renvoient désormais à cette édition.

5. Cf. J.-M. Shaeffer, Pourquoi la fiction ?, Paris, Seuil, 1999.

6. D. Viart, «Les "fictions critiques" dans la littérature contemporaine », in M. Majorano (dir.), Le goût du roman, Bari, Edizioni B.A. Graphis, 2002, p. 30-45. La notion a ensuite été reprise dans D. Viart, B. Vercier, La littérature française au présent. Héritage, modernité, mutations, Paris, Bordas, 2005 (1 ${ }^{\text {ère }}$ édition), 2008 ( 2 ème édition).

7. F. Jost, L'Cil caméra, Presses Universitaires de Lyon, 1987. 
8. Cf. G. Genette, Figures III, Paris, Seuil, 1972.

9. S. Rongier, A propos de Pas le bon pas le truand de Patrick Chatelier, remue.net, 10/07/2010. URL : http://remue.net/spip.php?article3736 : «La cinématière est ici à entendre comme un mode de relation esthétique et critique à l'image cinématographique. Elle devient un véritable matériau de travail, une matière d'image, un corps à la fois générique et inachevé produisant d'autres formes à partir d'un impensé de l'image [...] producteur de fantômes et donc de récits. » (Consulté le 15.10.2012)

10. Cf. J. Pouillon, Temps et roman, Paris, Gallimard, [1946], 1993.

\section{RÉSUMÉS}

Par-delà l'effet rebound, où le cinéma entre dans la littérature par la transposition de ses techniques scripturales et de composition, l'on assiste aujourd'hui à une véritable intégration des stylèmes et des topoï cinématographiques dans le roman. Par une analyse minutieuse de Pas le bon pas le truand de Patrick Chatelier, l'on interroge ici ce processus d'assimilation qui travaille la matière $d u$ texte du point de vue thématique et structural. L'écrivain démontre une grande attention aux structures créatives cinématographiques qu'il utilise pour composer son texte. À partir de la réécriture d'une séquence filmique de Sergio Leone, il assimile à sa matière textuelle deux procédés de création : le ralenti et la vision. Le ralenti permet de raconter les scènes dans les moindres détails, mais aussi de mimer le champ/contrechamp ; la primauté de l'aspect visuel dans la construction du récit met en scène une réalité en équilibre entre vrai et vraisemblable filmique.

Beyond the rebound effect, where the movie enters into the literature by transposition of scriptural and composition techniques, we are now witnessing a true integration of cinema stylèmes and topoï in the novel. By a deep analysis of Patrick Chatelier's Pas le bon pas le truand, we question this assimilation process that works the material of the text in terms of thematic and structural. The writer demonstrates attention to creative film structures and he uses it to compose his text. In rewriting a scene from a Sergio Leone film, he assimilates into his textual material two processes: the slowness with which the scenes are told in detail, and repeated so as to mimic the changes in visual perspective, the shot / reverse shot, and the primacy of the visual in the constructionof the narrative, which depicts a true balance between reality and film probability.

\section{INDEX}

Index géographique : France

Index chronologique : XXIe siècle

Mots-clés : cinématière, écriture cinématographique, ocularisation, Patrick Chatelier, roman, western 


\section{AUTEUR}

\section{ELISA BRICCO}

Université de Gênes 